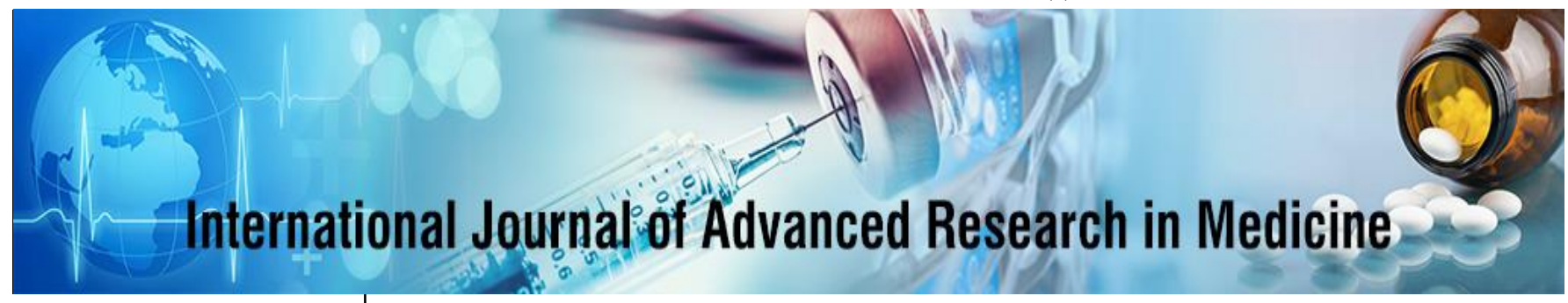

E-ISSN: 2706-9575

P-ISSN: 2706-9567

IJARM 2021; 3(2): 354-359

Received: 15-05-2021

Accepted: 29-06-2021

Dr. Venkatesh BC

Assistant Professor,

Department of Pulmonary

Medicine, Sapthagiri Institute

of Medical sciences, Bangalore,

Karnataka, India

Dr. Kiran N

Assistant Professor,

Department of Pulmonary

Medicine, Sapthagiri Institute

of Medical sciences, Bangalore,

Karnataka, India
Corresponding Author:

Dr. Kiran N

Assistant Professor,

Department of Pulmonary

Medicine, Sapthagiri Institute

of Medical sciences, Bangalore,

Karnataka, India

\title{
A study on correlation of Spirometry with obstructive sleep apnea
}

\section{Dr. Venkatesh BC and Dr. Kiran N}

DOI: https://doi.org/10.22271/27069567.2021.v3.i2f.268

\begin{abstract}
Relative proportions of sleep stages within a sleep cycle changes as the sleep period progresses. First cycle contains the greatest amount of stage 3 sleep. Episodes of slow wave sleep tend to be shorter in later sleep cycles. Also proportion of stage 3 sleep within slow wave sleep episodes diminishes as sleep period advances. The study was a prospective study conducted in the Department of Pulmonary Medicine, at a tertiary care hospital. Before proceeding for the study, the proforma and plan of the study were submitted to the Institutional Ethics committee for Research on Human Subjects and approval was obtained. Paired ' $t$ ' test was used to evaluate improvement after CPAP titration. The $p$ value $<0.05$, this was significant and showed that patients with OSA were benefitted from administration of CPAP. 10 out of 25 i.e. $40 \%$ of COPD patients were found to have OSA, 3 out of 12 i.e. $25 \%$ of bronchial Asthma patients and 2 out of 8 i.e. $25 \%$ of ILD patients were found to have OSA. None of the 5 patients of post infective fibrosis had OSA.
\end{abstract}

Keywords: Spirometry, Obstructive Sleep Apnea, CPAP

\section{Introduction}

Sleep in normal subject under normal condition, progresses through sleep stages in order from stage 1 to stage 3 followed by REM sleep. After onset of stage 1 sleep which lasts for few seconds to few minutes sleep progresses into stage 2, then transitions into slow wave sleep which leading into longer period of stage 3 sleep. Surprisingly however stage 2 repeats backwards before onset of REM sleep occurs. Each cycle throughout the sleep period lasts 90 to 120 minutes. So a normal sleep cycle has this pattern: waking $\rightarrow$ stage $1 \rightarrow 2 \rightarrow 3 \rightarrow$ $2 \rightarrow$ REM.

Progression of sleep stages can be disrupted at any time by an arousal that may cause shift to a lighter sleep stage or wakefulness. Arousals occur in response to intrinsic or extrinsic stimuli. Sleep disorders such as Obstructive Sleep Apnea or Periodic Limb Movements in sleep, causing increase frequency of arousals ${ }^{[1]}$.

Relative proportions of sleep stages within a sleep cycle changes as the sleep period progresses. First cycle contains the greatest amount of stage 3 sleep. Episodes of slow wave sleep tend to be shorter in later sleep cycles. Also proportion of stage 3 sleep within slow wave sleep episodes diminishes as sleep period advances.

First REM sleep onset occurs 90 minutes after initial sleep onset. Latency for onset of REM sleep decreases with advancing age. REM sleep periods are relatively brief in early sleep period, becoming longer towards morning. Early REM periods tend to have relatively brief periods of tonic REM sleep but these episodes increases in duration during later REM episodes ${ }^{[2]}$.

At birth, individuals sleep most of the time, but sleep periods of 1 to 4 hours distributed evenly throughout the day night. About half of sleep time is active (REM) Sleep, and half is quite (NREM) Sleep. Over the first year of life, sleep periods gradually lengthen, and proportion of REM sleep declines. By age of 6 months, 5 distinct sleep stages have developed. During early childhood, sleep begins to consolidate during nocturnal sleep period with periods of napping during the day. Percentage of REM sleep is highest during infancy and early childhood, drops of during adolescence and young adulthood, and decreases further in older age. By school age (6 to 12 years) napping declines and the relative proportion of REM sleep approaches that seen in adulthood. From birth through adolescence, the amount of slow-wave sleep decreases dramatically. This trend continues into adulthood and 
advanced age ${ }^{[3]}$.

During adulthood, many social and economic factors modify the timing and duration of sleep period, but it typically is consolidated into a single nocturnal sleep period of approximately 8 hour duration. With advancing age, total sleep time gradually declines, and onset of the primary sleep period advances.

OSA is a common condition that is characterized by repetitive episodes of closure and/or narrowing of upper airway reducing the airflow during sleep, leading to intermittent hypoxemia, arousal and sleep fragmentation ${ }^{[4]}$. Various global epidemiologic studies have demonstrated the prevalence of OSAS to vary from $0.3-5.1 \%$. These prevalent estimates, however, are based on data from predominantly white populations and may not be applicable to other racial groups. The prevalence of OSAS in adult Indian population is approximately 3.5\%. Major etiologic factors include obesity and craniofacial anatomic predisposition ${ }^{[5]}$. However, the majority of sleep apnoeics remain undiagnosed.

Conceptually, the upper airway is a compliant tube with lack of rigid supporting structure and, therefore, is subject to collapse ${ }^{[6]}$.

Critical closing pressure (PCRIT) is the trans-mural pressure (difference between the airway intraluminal pressure and the pressure exerted by surrounding tissues) required to collapse the upper airway. Pcrit is measured during sleep by changing intraluminal pressure (e.g., lowering CPAP level in an OSA patient or applying negative pressure in a normal subject) and assessing flow reductions. A more negative Pcrit denotes a less collapsible airway. Studies have shown a continuum of upper airway collapsibility in subjects with normal breathing (Pcrit $<-10 \mathrm{~cm} \mathrm{H} 2 \mathrm{O}$ ), nonapneic snoring (Pcrit range, -10 to $-5 \mathrm{~cm} \mathrm{H} 2 \mathrm{O}$ ), obstructive hypopnea (Pcrit range, -5 to $0 \mathrm{~cm} \mathrm{H} 2 \mathrm{O}$ ), and obstructive apnea (Pcrit $>0 \mathrm{~cm} \mathrm{H2O}$ ). These values are for "passive" Pcrit, which reflects the passive mechanical properties of the airway. Techniques are also available to measure "active" Pcrit, which reflects active neuromuscular compensation for reduced intraluminal pressure. Active Pcrit measurements demonstrate a significant impairment in neuromuscular compensation in OSA patients ${ }^{[7]}$.

Most patients with obstructive sleep apnea syndrome demonstrate upper airway obstruction at either the level of the soft palate (i.e. nasopharynx) or the level of the tongue (i.e. oropharynx). Research indicates that both anatomic and neuromuscular factors are important.

Anatomic factors (e.g. enlarged tonsils; volume of the tongue, soft tissue, or lateral pharyngeal walls; length of the soft palate; abnormal positioning of the maxilla and mandible) decrease the cross-sectional area of the upper airway and/or increase the pressure surrounding the airway, both of which predispose the airway to collapse ${ }^{[8]}$.

Upper airway neuromuscular activity, including reflex activity, decreases with sleep, and this decrease may be more pronounced in patients with OSAS ${ }^{[9]}$. Thus upper airway may be particularly vulnerable to collapse at the end of expiration. Reduced ventilatory motor output to upper airway muscles is believed to be the critical initiating event leading to upper airway obstruction; this effect is most pronounced in patients with an upper airway predisposed to collapse for anatomical reasons.

\section{Methodology}

The study was a prospective study conducted in the Department of Pulmonary Medicine, at a tertiary care hospital. Before proceeding for the study, the proforma and plan of the study were submitted to the Institutional Ethics committee for Research on Human Subjects and approval was obtained.

Patients with respiratory symptoms attending our OPD were selected randomly and explained about the study. They were screened by history and clinical examination. Investigations like chest X-ray, sputum examination (Gram's Stain and Smear for Acid Fast Bacilli), haematological tests, biochemical tests, computed tomography of thorax, Spirometry with reversibility testing and lung diffusion capacity test were conducted to establish the diagnosis of respiratory disorders like Chronic obstructive pulmonary disease (COPD), Bronchial asthma, Interstitial lung diseases (ILD), post infective fibrosis. After obtaining written, informed and valid consent, the patients were then subjected to Polysomnography study, followed by CPAP titration in those who diagnosed as OSA patients. The results obtained were analysed by The Statistical Package for the Social Sciences (SPSS-20) using the Pearson Chi-Square test, the Pearson Correlation test and paired ' $\mathrm{t}$ ' test. $\mathrm{p}$-value $<0.05$ denotes statistical significance.

The Methods adopted would be under following headings:

- Selection of subjects on the basis of inclusion and exclusion criteria

- Consent taking

- History and Clinical examination

- Investigations

\section{Selection of subjects}

The following were the inclusion and exclusion criteria for the subjects:

\section{Inclusion criteria}

1. Diagnosed patients of respiratory diseases i.e. Chronic obstructive pulmonary

2. disease (COPD), Bronchial asthma, Interstitial lung diseases (ILD), Post

3. infective fibrosis;

4. Patients aged above 18 years;

5. Patients of either gender.

\section{Exclusion criteria}

1. Patients with exacerbation of underlying respiratory disease;

2. Patients with Congestive cardiac failure;

3. Patients with Valvular heart disease;

4. Patients with Severe illnesses;

5. Patients with Renal failure;

6. Patients not willing to enrol for the study;

7. Patients who fit into these criteria were selected and explained the study;

\section{Results}

Based on Mallampati pharyngeal grade, out of total 50 patients, 31 had low risk of OSA (Mallampati pharyngeal grade 1 and 2) of these 4 had OSA and 19 had high risk of OSA (Mallampati pharyngeal grade 3 and 4) of these 11 had OSA. 
Table 1(a): Mallampati pharyngeal grading and OSA severity distribution

\begin{tabular}{|c|c|c|c|c|c|}
\hline OSA Severity & \multirow{2}{*}{ Normal } & Mild & Moderate & Severe & Total \\
\hline Mallampati & 27 & 1 & 2 & 1 & 31 \\
\hline 3 and 2 & 8 & 3 & 2 & 6 & 19 \\
\hline Total & 35 & 4 & 4 & 7 & 50 \\
\hline
\end{tabular}

Table 1(b): Statistical test results

\begin{tabular}{|c|c|c|}
\hline Test & Correlation coefficient ( r ) & p-value \\
\hline Pearson Chi-Square & -- & 0.005 \\
\hline Pearson Correlation & 0.513 & 0.000 \\
\hline
\end{tabular}

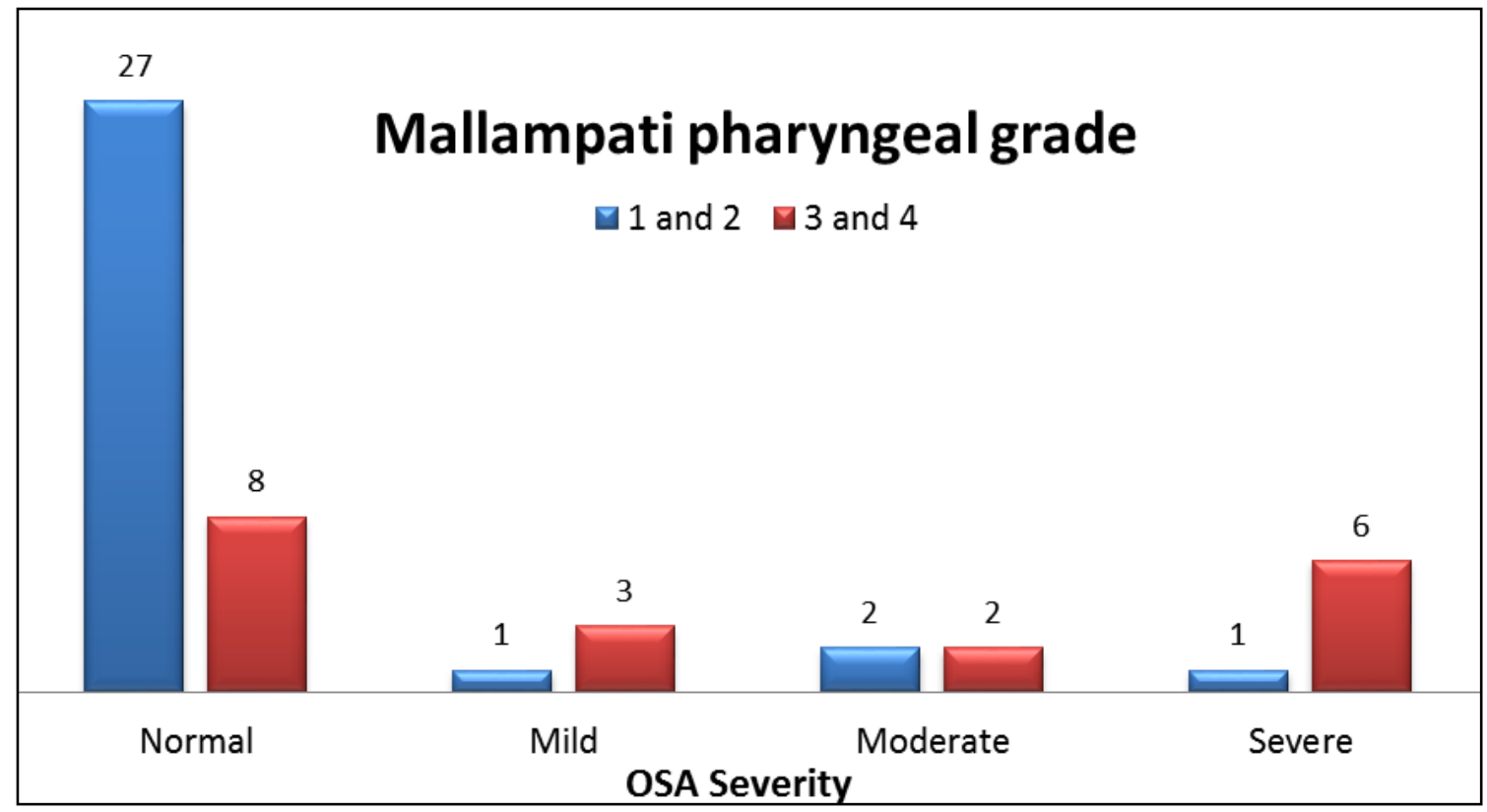

Graph 1: Mallampati pharyngeal grading and OSA severity distribution

Significant positive correlation was found between Mallampati pharyngeal grade and OSA severity. More, the Mallampati pharyngeal grade, more the severity of OSA.
Amongst 50 patients, 25 were of COPD, 12 were of Bronchial Asthma, 8 were of ILD and 5 were of Post infective fibrosis patients.

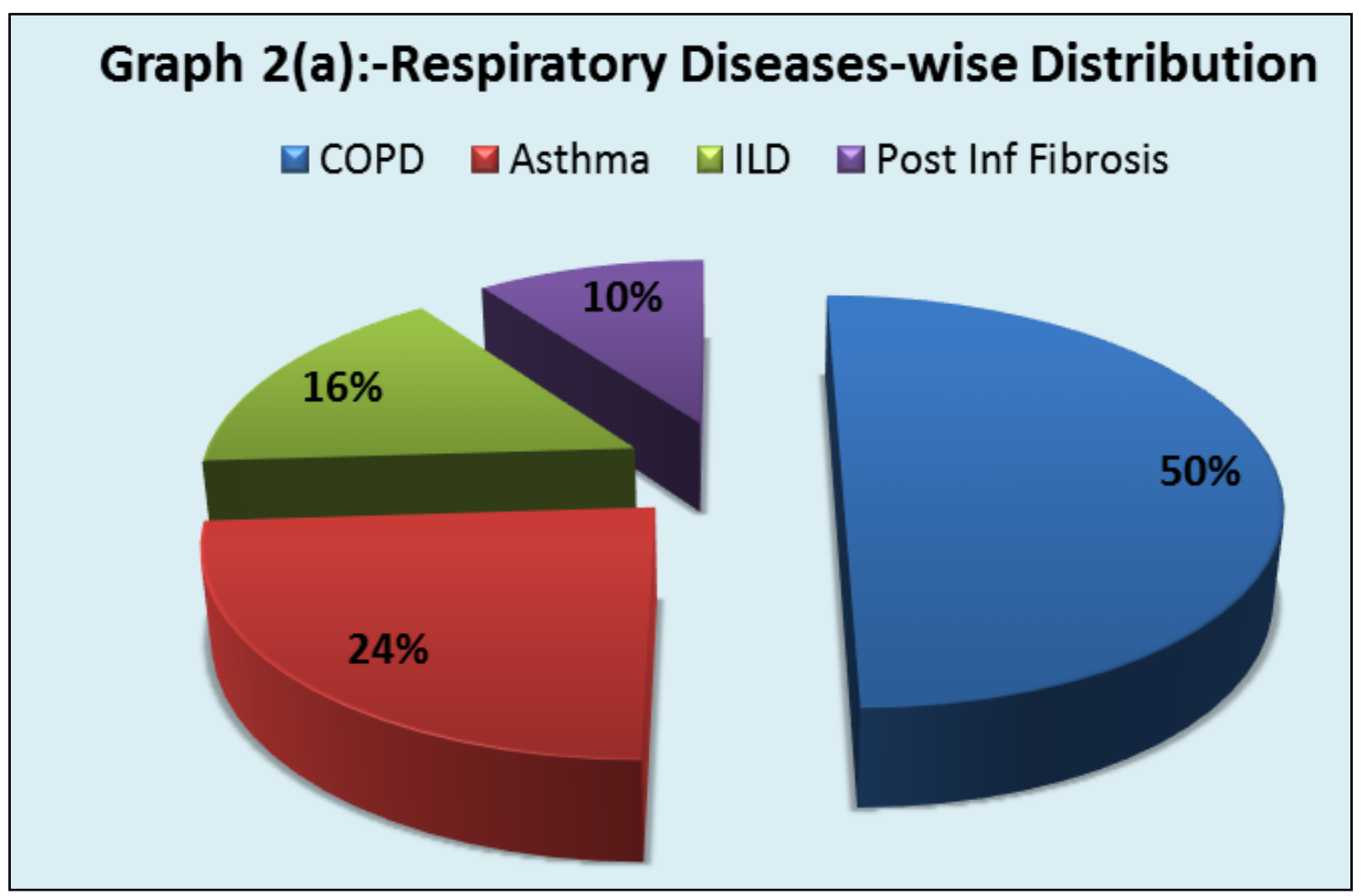

Graph 2 (c): Respiratory diseases and OSA severity distribution 
Table 2: Respiratory disorders and OSA severity distribution

\begin{tabular}{|c|c|c|c|c|c|}
\hline Diseases & \multirow{2}{*}{ Normal } & Mild & Moderate & Severe & Total \\
\hline COPD & 15 & 3 & 2 & 5 & 25 \\
\hline Asthma & 9 & 1 & 0 & 2 & 12 \\
\hline ILD & 6 & 0 & 2 & 0 & 8 \\
\hline PIF & 5 & 0 & 0 & 0 & 5 \\
\hline Total & 35 & 4 & 4 & 7 & 50 \\
\hline
\end{tabular}

Pearson Chi-Square test $\mathrm{p}$-value $=0.402$

Out of these patients, $10(40 \%)$ patients of COPD, 3(25\%) patients of Bronchial Asthma and 2(25\%) patient of ILD had OSA. None of the Post infective fibrosis patients had OSA.

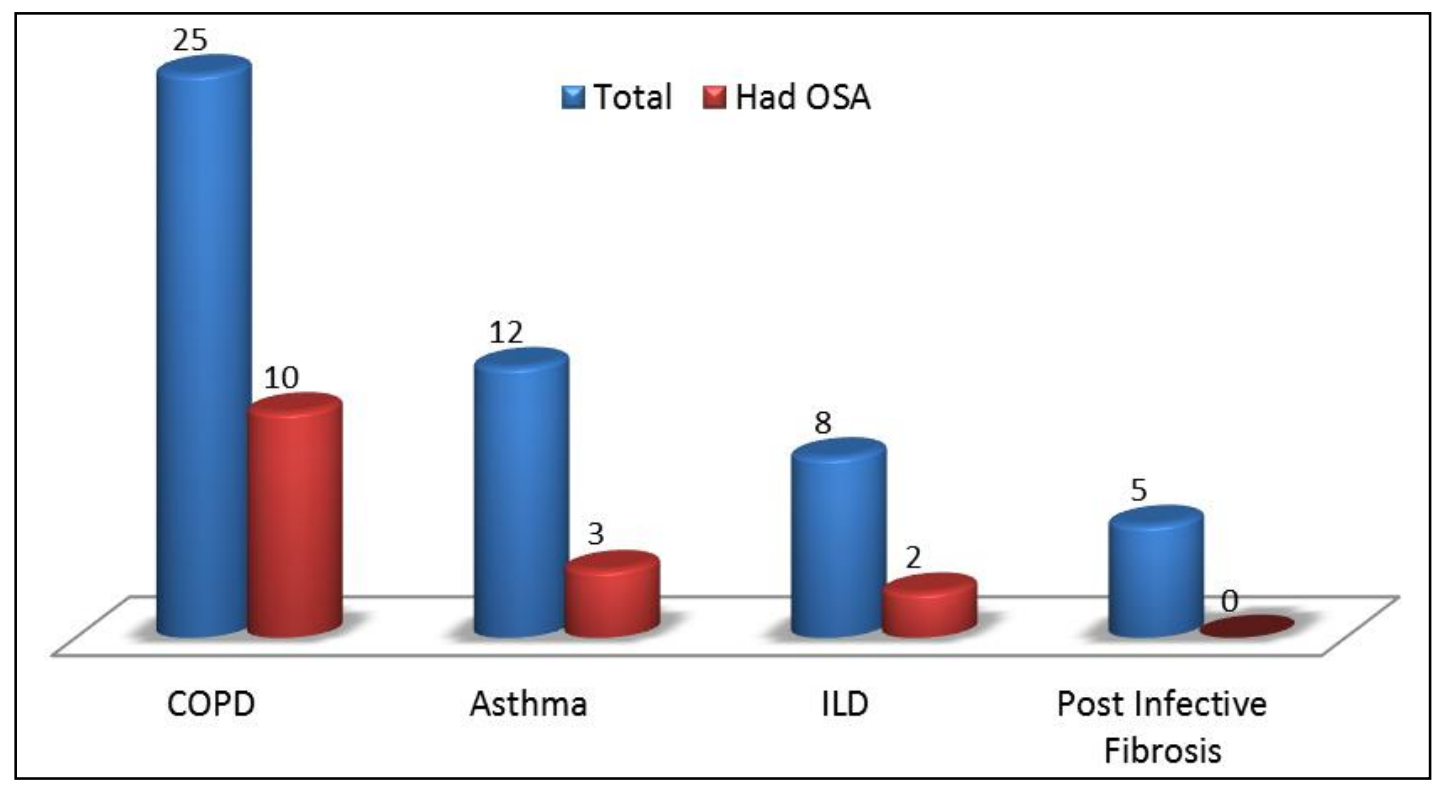

Graph 2(b): OSA prevalence in Respiratory Diseases

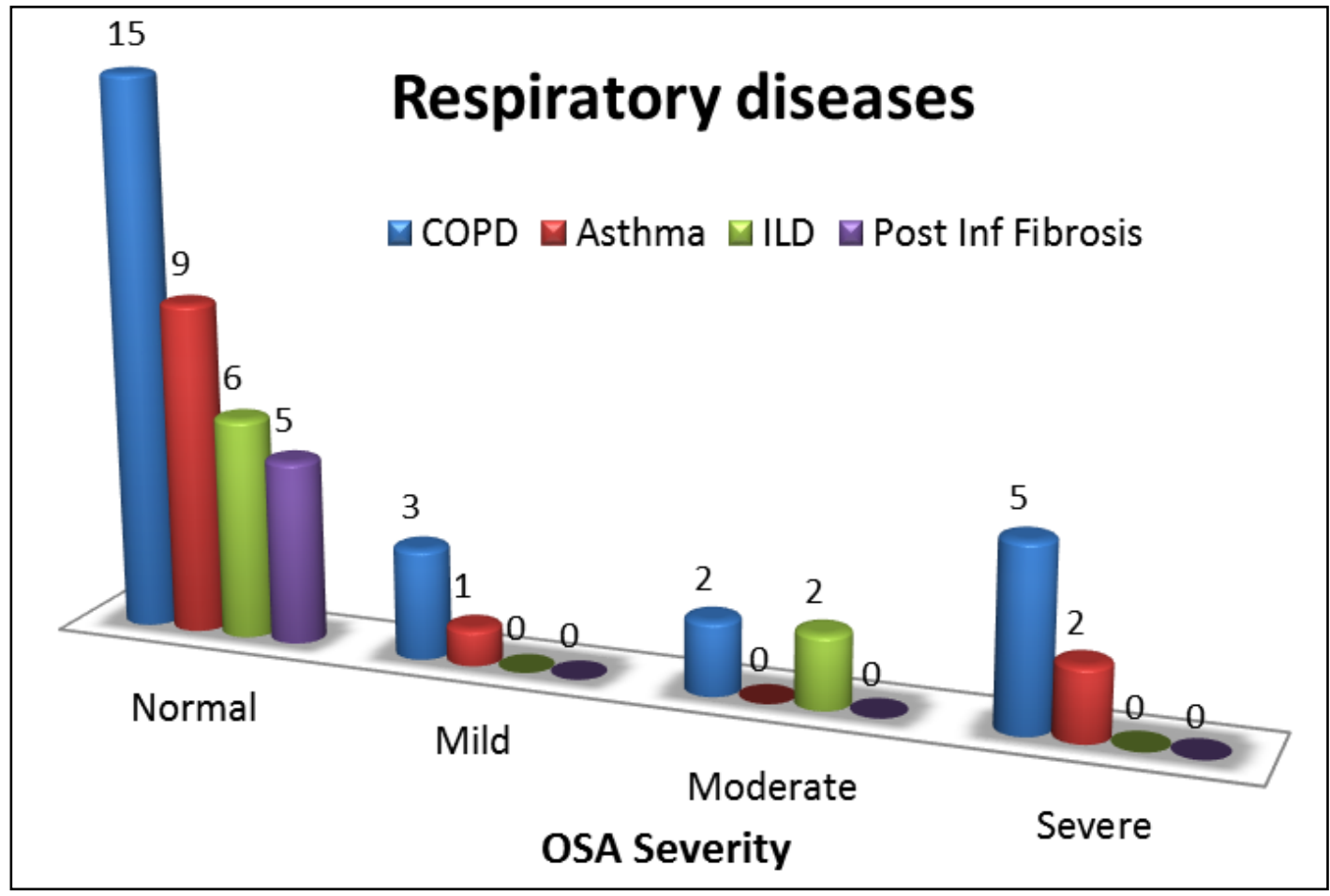

Graph 2 (c): Respiratory diseases and OSA severity distribution

Though the OSA was more common in COPD, there was no correlation between respiratory disorders and severity of OSA.
Amongst total 50 patients, 21 had FEV1\% vales $>65 \%, 17$ had FEV1\% values between $51-65 \%$ and 12 had FEV1\% values $<50 \%$. 
Table 3(a): FEV1\% and OSA severity distribution

\begin{tabular}{|c|c|c|c|c|c|}
\hline FEV1\% OSA Severity & \multirow{2}{*}{ Normal } & Mild & Moderate & Severe & Total \\
\hline$<50 \%$ & 8 & 1 & 2 & 1 & 12 \\
\hline $51-65 \%$ & 9 & 1 & 2 & 5 & 17 \\
\hline$>65 \%$ & 18 & 2 & 0 & 1 & 21 \\
\hline Total & 35 & 4 & 4 & 7 & 50 \\
\hline
\end{tabular}

Table 3(b): Statistical test results

\begin{tabular}{|c|c|c|}
\hline Test & Correlation coefficient ( r ) & p-value \\
\hline Pearson Chi-Square & -- & 0.164 \\
\hline Pearson Correlation & -0.128 & 0.374 \\
\hline
\end{tabular}

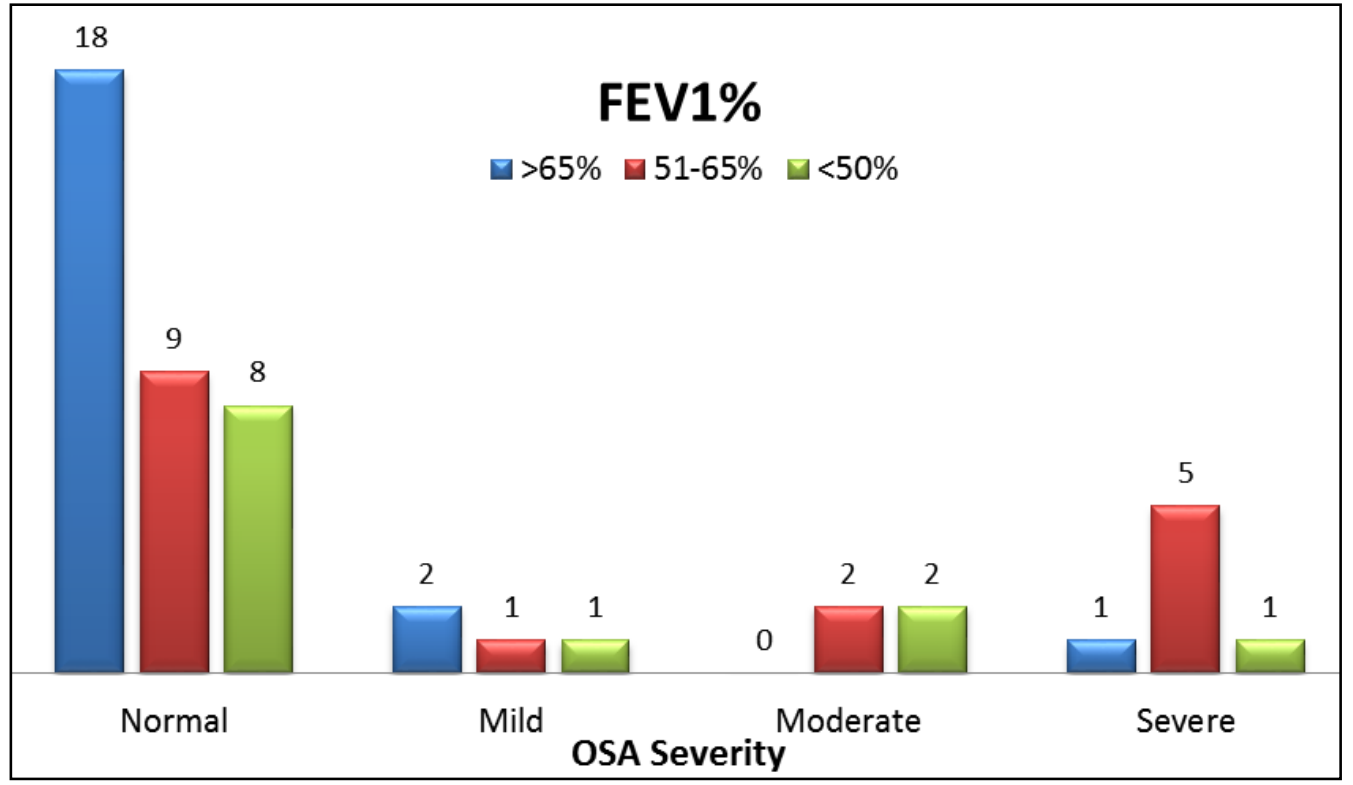

Graph 3: FEV1\% and OSA severity distribution

There was no correlation between FEV1\% values and severity of OSA.

Out of 50, those 15 patients who had OSA underwent overnight CPAP titration. It was noted that average AHI before CPAP was $31.70 / \mathrm{hr}$., after administration of CPAP (average CPAP is $11.26 \mathrm{~cm}$ of $\mathrm{H}_{2} \mathrm{O}$ ), average AHI was $2.80 / \mathrm{hr}$.

Table 4: Result of CPAP

\begin{tabular}{|c|c|}
\hline CPAP & Average AHI \\
\hline Before CPAP & $31.70 / \mathrm{hr}$. \\
\hline After CPAP & $2.80 / \mathrm{hr}$. \\
\hline
\end{tabular}

p-value $=<0.05$

Paired ' $t$ ' test was used to evaluate improvement after CPAP titration. The $\mathrm{p}$-value $<0.05$, this was significant and showed that patients with OSA were benefitted from administration of CPAP.

\section{Discussion}

The study was to assess obstructive sleep Apnea (OSA) in patients with Respiratory disorders like chronic obstructive pulmonary disease (COPD), Bronchial asthma, interstitial lung diseases (ILD) \& Post infective fibrosis and to find the treatment outcome after CPAP titration in patients with OSA.

In our study, patients attending the out-patient department (OPD) of pulmonary medicine were screened for symptoms, clinical history was documented. Clinical examination, relevant investigations i.e. blood investigations (Haemoglobin, CBC, fasting and post prandial Blood sugar levels, lipid profile, Thyroid profile, etc.), sputum examination (in relevant cases), ECG and 2D Echo, Chest $\mathrm{X}$-ray, CT/HRCT, Spirometry were performed respectively. The patients diagnosed with respiratory disorders were subjected to polysomnography. CPAP titration was carried out in patients who diagnosed as OSA. The youngest patient was 28 years old and the oldest was 70 years old, mean age was 51 years. $62 \%$ of the patients were males and $38 \%$, females. In our study of 50 patients with respiratory disorders, 25 were of COPD, 12 of Bronchial Asthma, 8 of ILD and 5 with Post infective fibrosis. Based on the polysomnography report out of 50 patients, $15(30 \%)$ were found to have Obstructive Sleep Apnea. The comorbidities (Diabetes mellitus, Hypertension, Ischaemic heart disease and Hypercholesterolemia) were observed in group who had OSA. Out of these patients, 10 out of $25(40 \%)$ patients of COPD, 3 out of $12(25 \%)$ patients of Bronchial Asthma and 2 out of $8(25 \%)$ patients of ILD had OSA. None of the Post infective fibrosis patients had OSA.

In our study Mallampati pharyngeal grade was found to be positively correlates with severity of OSA ( $\mathrm{r}=0.513$, $\mathrm{p}<0.05)$. Myers KA, Mrkobrada M, et al. ${ }^{[10]}$ found similar findings.

Amongst 50 patients of pre-existing respiratory diseases, 15 found to have OSA i.e. $30 \%$. $40 \%$ of COPD patients, $25 \%$ of Bronchial Asthma and $25 \%$ of ILD patients had OSA. None of the Post infective fibrosis patients had OSA. In the 
studies conducted by Lopez-Acevedo MN, Torres-Palacios A, et al. ${ }^{[11]}$ and Sridhar Venkateswaran, Augustine Tee ${ }^{[12]}$ found that $10-20 \%$ of COPD and 63\% COPD patients had OSA, Mermigkis C, Stagaki E found 59\% of IPF patients had OSA and Hany Shaarawy, Nasr Affara ${ }^{[13]}$ found $25 \%$ of the Bronchial Asthma patients had OSA.

In our study we found that there was no significant correlation between FEV1 and AHI (p value 0.374). Though OSA was common in group with FEV1 51-65\%, it was not found to be statistically significant. These findings are in accordance with the studies conducted by Bertrand Herer, MD; Nicolas Roche, MD, et al. ${ }^{[14]}$, Bhavneesh Sharma, Steven Feinsilver, et al. ${ }^{[15]}$ and M. Harish, R. Shine Shukoor, et al. ${ }^{[16]}$.

In our study there was significant reduction in AHI after overnight CPAP titration $(\mathrm{p}<0.05)$. The studies conducted by McDaid C, Griffin S, ${ }^{[17]}$ and Stammnitz A, Jerrentrup A, et al. ${ }^{[18]}$ found that CPAP is an effective treatment for OSA compared with conservative/usual care and placebo. However the findings of this study need to be confirmed by field study.

\section{Conclusion}

- Significant positive correlation was found between Mallampati pharyngeal grade and severity of OSA.

- There was no correlation between FEV1\% and severity of OSA.

- $\quad$ CPAP is the effective treatment modality of OSA (i.e. it reduces the Apnea Hypopnea Index).

- $30 \%$ of the patients with respiratory disorders (COPD, bronchial Asthma and ILD) included in our study had OSA. It was observed that none of the patients with post infective fibrosis had OSA.

\section{References}

1. Osler W. The Principles and Practice of Medicine, 8th ed., New York, NY and London: Appleton \& Co, 1918.

2. Bickelmann AG, Burwell CS, Robin ED, Whaley RD. Extreme obesity associated with alveolar hypoventilation: A Pickwickian syndrome. Am J Med. 1956;21(5):811-8.

3. Gastaut H, Tassinari CA, Duron B. Polygraphic study of diurnal and nocturnal (hypoid and respiratory) Episodal manifestations of Pickwick syndrome Rev Neurol (Paris). 1965;112(6):568-79.

4. Jung R, Kuhlo W. Neurophysiological studies of abnormal night sleep and the pickwickian syndrome, Prog Brain Res. 1965;18:140-59.

5. Kuhlo W, Doll E, Franck MC. Successful management of Pickwickian syndrome using long-term tracheostomy Dtsch Med Wochenschr. 1969;94(24):1286-1290.

6. Sullivan CE, Issa FG, Berthon-Jones M, Eves L. Reversal of obstructive sleep apnoea by continuous positive airway pressure applied through the nares, Lancet. 1981;1(8225):862-5.

7. Fujita S, Conway W, Zorick F, Roth T. Surgical correction of anatomic abnormalities in obstructive sleep apnea syndrome: Uvulopalatopharyngoplasty. Otolaryngol Head Neck Surg. 1981; 89(6):923-34.

8. Silber MH, Ancoli-Israel S, Bonnet MH, Chokroverty $\mathrm{S}$, Grigg-Damberger MM, Hirshkowitz $\mathrm{M}$, et al. (March). "The visual scoring of sleep in adults", Journal of Clinical Sleep Medicine. PMID 17557422. 2007;3(2):121-31.
9. Schwartz WJ, Stakes JW, Martin JB. The sleep-wake cycle and disorders of sleep. In Braunwald, et al., eds. Harrison's principles of internal medicine. New York: McGraw-Hill, 1987, 111.

10. Myers KA, Mrkobrada M, Simel D. Does This Patient Have Obstructive Sleep Apnea? The Rational Clinical Examination, Systematic Review JAMA 2013;310(7):731-741.

11. Lopez-Acevedo MN, Torres-Palacios A, Elena OcasioTascon M, Campos-Santiago Z, Rodriguez-Cintron W. Overlap syndrome: An indication for sleep studies? : A pilot study. Sleep \& breathing $=$ Schlaf \& Atmung. 2009;13(4):409-13.

12. Sridhar Venkateswaran, Augustine Tee. Overlap syndrome between chronic obstructive pulmonary disease and obstructive sleep apnoea in a Southeast Asian Teaching Hospital Singapore Med J. 2014;55(9):488-492.

13. Hany Shaarawy, Nasr Affara. Assessment of the prevalence of obstructive sleep apnea in patients with stable uncontrolled asthma, impact of continuous positive airway pressure treatment Egyptian, Journal of Chest Diseases and Tuberculosis. 2013;62:183-187.

14. Bertrand Herer MD, Nicolas Roche MD, et al. Value of Clinical, Functional, and Oximetric Data for the Prediction of Obstructive Sleep Apnea in Obese Patients, CHEST. 1999;116:1537-1544.

15. Bhavneesh Sharma, Steven Feinsilver, et al. Obstructive airway disease and obstructive sleep apnea: Effect of pulmonary function Lung. 2011;189(1):37-4.

16. M Harish, R Shine Shukoor, et al. Comparative study of sleep apnoea In chronic bronchitis and Emphysema, World J. Med. Sci. 2014;10(1):50-55.

17. McDaid C, Griffin S, Weatherly H, Durée K, Van der Burgt M, Van Hout S, et al. Continuous positive airway pressure devices for the treatment of obstructive sleep apnoea-hypopnoea syndrome: A systematic review and economic analysis. Health Technol Assess., XI-XIV, 1119,143-274. 2009;13(4):III-IV.

18. Stammnitz A, Jerrentrup A, Penzel T, Peter JH, Vogelmeier C, Becker HF. Automatic CPAP titration with different self-setting devices in patients with obstructive sleep Apnoea Eur Respir J. 2004;24(2):2738 . 